\title{
Fractal analysis of shape factor for matrix-fracture transfer function in fractured reservoirs
}

\author{
Lan $\mathrm{Mei}^{1}$, Heng Zhang ${ }^{1, *}$, Lei Wang ${ }^{1}$, Qi Zhang ${ }^{1}$, and Jianchao Cai ${ }^{1,2,{ }^{*}}$ \\ ${ }^{1}$ Key Laboratory of Tectonics and Petroleum Resources, Ministry of Education, China University of Geosciences, \\ Wuhan 430074, PR China \\ ${ }^{2}$ Institute of Geophysics and Geomatics, China University of Geosciences, Wuhan 430074, PR China
}

Received: 17 February 2020 / Accepted: 27 May 2020

\begin{abstract}
As the core function of dual-porosity model in fluids flow simulation of fractured reservoirs, matrixfracture transfer function is affected by several key parameters, such as shape factor. However, modeling the shape factor based on Euclidean geometry theory is hard to characterize the complexity of pore structures. Microscopic pore structures could be well characterized by fractal geometry theory. In this study, the separation variable method and Bessel function are applied to solve the single-phase fractal pressure diffusion equation, and then the obtained analytical solution is used to deduce one-dimensional, two-dimensional and three-dimensional fractal shape factors. The proposed fractal shape factor can be used to explain the influence of microstructure of matrix on the fluid exchange rate between matrix and fracture, and is verified by numerical simulation. Results of sensitivity analysis indicate that shape factor decreases with tortuosity fractal dimension and characteristic length of matrix, increases with maximum pore diameter of matrix. Furthermore, the proposed fractal shape factor is effective in the condition that tortuosity fractal dimension of matrix is roughly between 1 and 1.25. This study shows that microscopic pore structures have an important effect on fluid transfer between matrix and fracture, which further improves the study on flow characteristics in fractured systems.
\end{abstract}

$\lambda_{\max }$
$A_{0}$
$c$
$t$
$R$

Maximum pore diameter of matrix, [L] Unit area, $\left[\mathrm{L}^{2}\right]$

Total compressibility of reservoir, $\left[\left(\mathrm{LT}^{2}\right) / \mathrm{M}\right]$

Transfer flow time, [T]

Equivalent radius, [L]

\section{Nomenclature}

$q$

$\sigma$

$\rho$

$\mu$

$N$

$L$

$P$

$P_{0}$

$\bar{P}$

$P_{f}$

$K_{m}$

$\phi_{m}$

$K_{a}$

$\phi_{a}$

$D_{f}$

$D_{T}$ fracture, $\left[\mathrm{M} /\left(\mathrm{TL}^{3}\right)\right]$

Shape factor, $\left[1 / \mathrm{L}^{2}\right]$

Fluid density, $\left[\mathrm{M} / \mathrm{L}^{3}\right]$

Fluid viscosity, [M/(LT)]

Number of sets of fractures $(1,2$ or 3$)$

Characteristic length of matrix, [L]

Matrix pressure, [M/(LT $\left.\left.{ }^{2}\right)\right]$

Initial pressure of matrix, $\left[\mathrm{M} /\left(\mathrm{LT}^{2}\right)\right]$

Average pressure of matrix, $\left[\mathrm{M} /\left(\mathrm{LT}^{2}\right)\right]$

Fracture pressure, $\left[\mathrm{M} /\left(\mathrm{LT}^{2}\right)\right]$

Matrix permeability, $\left[\mathrm{L}^{2}\right]$

Matrix porosity

Permeability constant, $\left[\mathrm{L}^{2}\right]$

Porosity constant

Fractal dimension of matrix

Tortuosity fractal dimension of matrix

\footnotetext{
* Corresponding authors: cughzhang@163.com, caijc@cug.edu.cn
}

\section{Introduction}

Naturally fractured reservoirs account for a large proportion of the world's resources and play an important role in the world's energy structure. Unconventional resources have significantly transformed the landscape of oil and gas industry in these years. Hydraulic fracturing is a primary technology for economical and effective exploitation of unconventional reservoirs (Li et al., 2015; Wang et al., 2017; Behnoudfar et al., 2019; Vishkai and Gates, 2019), which aims at creating large-scale fracture network to increase production. Therefore, fluids flow mechanism in fractured systems has aroused the strong interest of petroleum engineers, geothermal engineers and geologists, etc.

The dual-porosity model is one of common simulation methods to study the flow properties in fractured reservoirs. 
The concept of dual-porosity media in fractured reservoirs was firstly proposed by Barenblatt et al. (1960), and then was applied by Warren and Root (1963) to the field of petroleum engineering. As shown in Figure 1, fractured reservoirs are usually supposed to consist of a continuous fracture region that serves as the primary flow channels (high permeability and low porosity), and a discontinuous matrix region which acts as the primary hydrocarbon storage area (low permeability and high porosity). They hypothesized that single-phase of slightly compressible fluid transfer between matrix and fracture occurred under pseudo-steady state conditions. The transfer function was originally formulated as (Warren and Root, 1963):

$$
q=\sigma \frac{\rho K_{m}}{\mu}\left(\bar{P}-P_{f}\right)
$$

where $q$ is the fluid transfer rate between matrix and fracture, $\left[\mathrm{M} /\left(\mathrm{TL}^{3}\right)\right] ; \sigma$ is the shape factor, $\left[1 / \mathrm{L}^{2}\right] ; \rho$ is the fluid density, $\left[\mathrm{M} / \mathrm{L}^{3}\right] ; \mu$ is the fluid viscosity, $[\mathrm{M} /(\mathrm{LT})] ; K_{m}$ is the matrix permeability, $\left[\mathrm{L}^{2}\right] ; \bar{P}$ is the average pressure of matrix, $\left[\mathrm{M} /\left(\mathrm{LT}^{2}\right)\right] ; P_{f}$ is the fracture pressure, $\left[\mathrm{M} /\left(\mathrm{LT}^{2}\right)\right]$.

The shape factor model for cubic matrix can be written as (Warren and Root, 1963):

$$
\sigma=\frac{4 N(N+2)}{L^{2}}
$$

where $N$ is the number of sets of fractures $(1,2$ or 3$), L$ is the characteristic length of matrix which is the distance between the fracture surface and the center of matrix, [L]. The shape factors are $12 / L^{2}, 32 / L^{2}$ and $60 / L^{2}$ for one-dimensional (1D), two-dimensional (2D) and threedimensional (3D) spaces, respectively.

Furthermore, many scholars have carried out studies on fluids exchange between matrix and fracture based on Warren and Root (1963)'s model. Kazemi et al. (1976) directly extended single-phase transfer of Warren and Root (1963) to two-phase transfer and firstly applied it to the numerical simulation of 3D isotropic dual-porosity porous media. When the length of matrix is the same in each direction, the shape factors are $4 / L^{2}, 8 / L^{2}$ and $12 / L^{2}$ for $1 \mathrm{D}, 2 \mathrm{D}$ and 3D spaces, respectively. By including the effects of gravity, capillary force and viscous force on transfer function, Thomas et al. (1983) established a 3D three-phase model to simulate fluids flow in fractured reservoirs. By comparing single-porosity fine-grid with the dual-porosity models, they concluded that shape factor was $25 / L^{2}$ when the two models have good agreement. Coats (1989) solved the diffusion equation of the slightly compressible fluids and derived a transfer function. They found that their shape factor was exactly twice the value of Kazemi et al. (1976). By comparing fine-grid models with experiments, Ueda et al. (1989) believed that the shape factor was twice and three times that of Kazemi et al. (1976) in 1D and 2D spaces, respectively.

However, the application of these shape factors to explain the transient characteristics of matrix pressure in the initial stage of fluids transfer process usually produces large deviation. Therefore, some scholars have conducted many studies on the description of matrix pressure transient behavior. Zimmerman et al. (1993) developed a single-phase dual-porosity model in fractured reservoirs and solved the non-linear ordinary differential equation to obtain matrix-fracture transfer function, which was more accurate than the linear Warren and Root (1963) equation to calculate the flux in the early and late stages. Lim and Aziz (1995) derived shape factor by solving matrix pressure diffusion equation, and used fine-grid numerical simulation to validate the presented shape factor. They considered that both the geometric of matrix and pressure gradient of the whole system have a great impact on shape factor. Noetinger and Estebenet (2000) used continuous time random walks methods to simulate matrix-fracture exchange

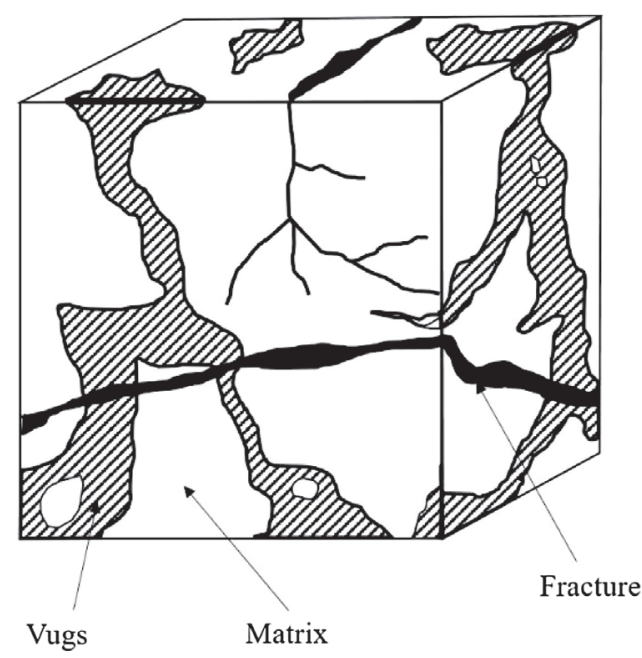

(a) Actual reservoir

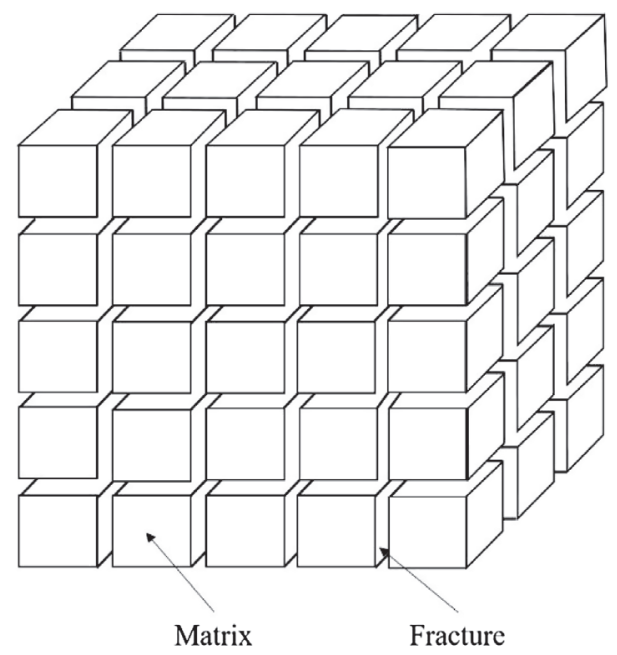

(b) Idealized model reservoir

Fig. 1. Schematic of idealization of fractured reservoirs (Warren and Root, 1963). 
term and obtained shape factor involved in dual-porosity formulations of fluid flow through fractured reservoirs. Good agreement were found between their results and numerical simulation. Sarma and Aziz (2004) believed that fracture networks were non-orthogonal, and solved the nonorthogonal single-phase pressure diffusion equation to deduce $2 \mathrm{D}$ and $3 \mathrm{D}$ shape factors. Their results were verified by comparing the discrete fracture model with the dualporosity model. For different geometries and boundary conditions, Hassanzadeh and Pooladidarvish (2006) introduced the shape factor, and indicated that both the geometric of matrix and pressure change of fracture have an important effect on the shape factor. Ranjbar et al. (2011) solved the single-phase nonlinear diffusivity equation of different pressure depletion regimes to derive the shape factor, and investigated the impact of fracture pressure depletion regime on the shape factor. They further applied the finegrid numerical simulation technique to verify their shape factor. By solving the saturation diffusion equation in fractured reservoirs, Saboorian-Jooybari et al. (2015) developed the shape factor accounting for both capillary and gravity forces, which was verified using fine-grid numerical simulation. For low permeability fractured reservoirs, He et al. (2017) proposed a new shape factor by solving single-phase pressure diffusion equation. Furthermore, their results shown that the new shape factor could predict production accurately. For tight fractured reservoirs with the existence of the boundary layer and the heterogeneous pressure distribution of matrix, Cao et al. (2019) presented the single-phase matrix-fracture transfer function, which was verified by the numerical simulation and the experimental data.

Fractal geometry theory has been widely used to study the transport properties of porous media. The application of fractal geometry theory in the field of petroleum engineering has received extensive attentions. Both of fracture network (Chang and Yortsos, 1990; Acuna et al., 1995) and matrix (Katz and Thompson, 1985; Krohn, 1988; Adler, 1996) can be represented effectively and conveniently by fractal geometry. Zhou et al. (2000) studied the fractal characteristics of natural fractures, and developed a fractal transfer function model to analyze the effect of pore size distribution of matrix on the fluids transfer between matrix and fracture in fractured reservoirs. Based on the fractal characteristics of fractured reservoirs, Flamenco-Lopez and Camacho-Velazquez (2001) established the fractal dual-porosity equation, and obtained the analytical solution of pressure by Laplace transform method. The importance of transient and pseudo-steady pressure characteristics of fractured reservoirs with fractal geometry in a single-well was discussed. Kong et al. (2009) described the pore characteristics of porous and fractured media through fractal theory and presented the new flow velocity, porosity and permeability models in both porous and fractured media. In their study, the pressure diffusion equations of porous and fractured media were established and their transient pressure characteristics were analyzed. Yao et al. (2012) believed that fractures have fractal characteristics and established two dual-porosity equations based on circular matrix and cylindrical matrix, respectively. They used Laplace method to get the pressure analytical solution, and analyzed the influences of matrix shape and fractal parameters on the transient pressure characteristics of fractured reservoirs. Fan and Ettehadtavakkol (2017) indicated that fractures have fractal characteristics and established induced fractures network distribution model, which was a function of density distribution and permeability/porosity distribution of induced fractures. Lian et al. (2018) used fractal geometry to characterize the different scale distribution of pores and fractures, and presented single-phase radial fluid flow equation by involving nonequilibrium adsorption to study the flow properties in shale gas reservoirs. Wang et al. (2018) combined the dual porosity model with the tri-linear flow model, and used fractal geometry to characterize the heterogeneous distribution of fracture networks and the non-uniform of both matrix/fracture porosity and permeability. By applying Bessel Function and Laplace transform techniques, the flow of fluid through primary hydraulic fractures, stimulated-reservoir-volume and unstimulated reservoir matrices were integrated to derive analytical solutions. The accuracy of the analytical solutions in their study were verified by a synthetic fine-grid numerical simulation example.

In the previous literature, the effect of matrix microstructure on the shape factor is not considered during reservoir development. Fractal geometry theory can relatively accurately characterize the effect of matrix microstructure on fluids transfer between matrix and fracture. Fractal geometry theory has been widely applied in flow characteristics of fractured reservoirs, but its applications in the transfer function and shape factor are still rare. In this paper, we consider the effect of matrix microstructure on fluids transfer between matrix and fracture by means of fractal geometry theory, from which a new shape factor is derived. Single-porosity fine-grid and dual-porosity models are then applied to validate the new shape factor. Finally, the influences of microstructure and characteristic length of matrix on the shape factor are accordingly discussed.

\section{Mathematical model}

It has been reported that pore surface and pore size distribution of porous media follow fractal features (Katz and Thompson, 1985; Yu and Cheng, 2002). The fractal geometry theory has great advantages in accurate representation of porous media with complex microstructures (Costa, 2006; Erol et al., 2017). Considering the fractal capillary bundle model and Hagen-Poiseuille equation of curved capillary, the fractal permeability and fractal porosity of matrix can be respectively expressed by (Kong et al., 2007):

$$
\begin{aligned}
K_{m} & =\frac{\pi}{128 A_{0}} \frac{D_{f} \lambda_{\max }^{4}}{D_{T}\left(3+D_{T}-D_{f}\right)}\left(\frac{x}{\lambda_{\max }}\right)^{1-D_{T}} \\
& =K_{a}\left(\frac{x}{\lambda_{\max }}\right)^{1-D_{T}}
\end{aligned}
$$




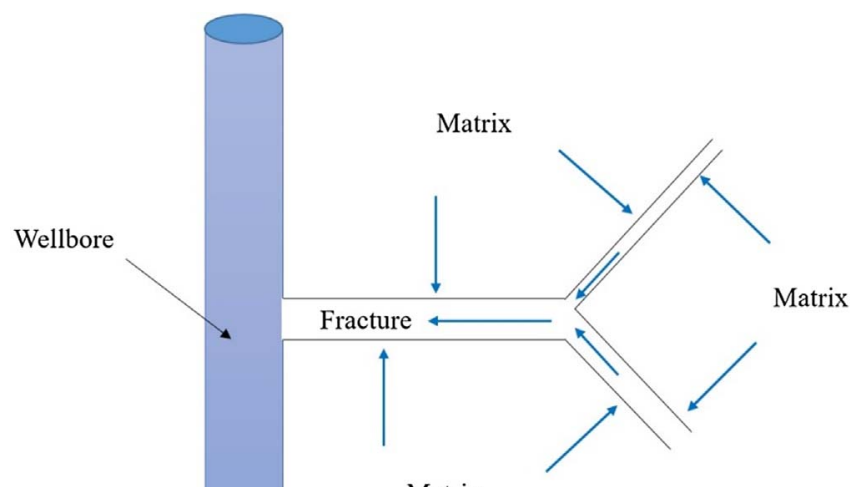

Matrix

Fig. 2. Schematic of dual-porosity media.

$$
\begin{aligned}
\phi_{m} & =\frac{\pi}{4 A_{0}} \frac{D_{T} D_{f} \lambda_{\max }^{2}}{3-D_{T}-D_{f}}\left[1-\left(\frac{\lambda_{\min }}{\lambda_{\max }}\right)^{3-D_{T}-D_{f}}\right]\left(\frac{\lambda_{\max }}{x}\right)^{1-D_{T}} \\
& =\phi_{a}\left(\frac{\lambda_{\max }}{x}\right)^{1-D_{T}}
\end{aligned}
$$

where $\phi_{m}$ is the matrix porosity; $A_{0}$ is the unit area, $\left[\mathrm{L}^{2}\right] ; x$ is the distance, $[\mathrm{L}] ; D_{f}$ is the pore fractal dimension, $0<D_{f}<2$ (in 2D space) and $0<D_{f}<3$ (in 3D space); $\lambda_{\max }$ is the maximum pore diameter, $[\mathrm{L}] ; D_{T}$ is the tortuosity fractal dimension, $1<D_{T}<2$ (in 2D space) and $1<D_{T}<3$ (in $3 \mathrm{D}$ space), $D_{T}=1$ represents a straight capillary, $D_{T}=2$ represents a plane is completely filled with tortuous line, and $D_{T}=3$ represents a $3 \mathrm{D}$ space is completely filled with tortuous line; $K_{a}$ is the permeability constant, $\left[\mathrm{L}^{2}\right] ; \phi_{a}$ is the porosity constant. It is noted that the symbols $D_{f}$ and $D_{T}$ are respectively used for pores and streamlines in matrix in this work.

Substituting equations (3) and (4) into the continuity equation, the matrix fractal pressure diffusion equation can be obtained by (Kong et al., 2007):

$$
\frac{\partial^{2} P}{\partial x^{2}}+\frac{\xi-D_{T}}{x} \frac{\partial P}{\partial x}=\frac{\phi_{a o} \mu c}{K_{a}}\left(\frac{x}{\lambda_{\max }}\right)^{2\left(D_{T}-1\right)} \frac{\partial P}{\partial t},
$$

where $\xi$ corresponds to $1 \mathrm{D}$ flow, 2D flow and 3D flow, which are 1,2 and 3, respectively. When the capillary is straight, equation (5) can be simplified as the classical pressure diffusion equation.

Before establishing mathematical model, some assumptions need to be considered as follows:

1. The fractured reservoirs are seen as a dual-porosity model (Fig. 2), where fluids in matrix flow through fractures instead of directly to the bottom of well, and matrix is isotropic.

2. The fluid is slightly compressible.

3. The rock is compressible.

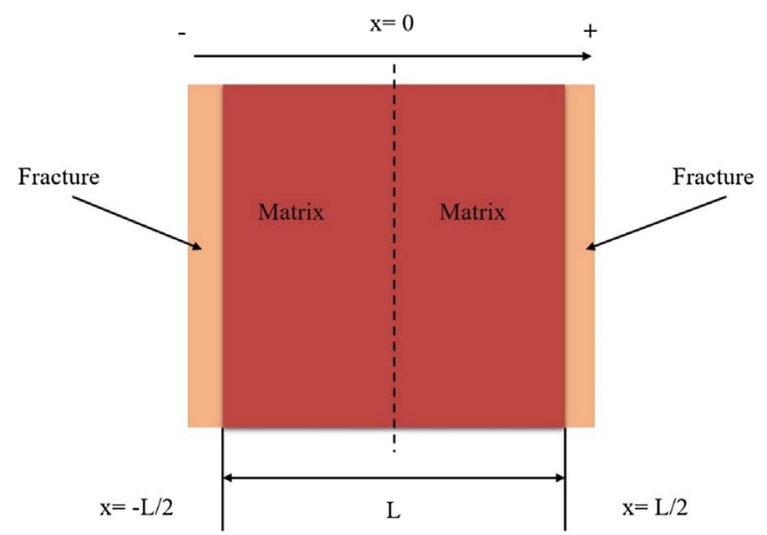

Fig. 3. Schematic of 1D matrix-fracture system.

4. The matrix is represented by the fractal tortuous capillary bundle model.

\subsection{Transfer function and shape factor of 1D flow}

Supposing matrix has been surrounded by two fractures, which have a spacing $L$ (Fig. 3), fluids flow from matrix to fracture surface, and therefore the fractal pressure diffusion equation in matrix can be given by Kong et al. (2007):

$$
\frac{\partial^{2} P}{\partial x^{2}}+\frac{1-D_{T}}{x} \frac{\partial P}{\partial x}=\frac{\phi_{a o} \mu c}{K_{a}}\left(\frac{x}{\lambda_{\max }}\right)^{2\left(D_{T}-1\right)} \frac{\partial P}{\partial t},
$$

where $P$ is the matrix pressure, $\left[\mathrm{M} /\left(\mathrm{LT}^{2}\right)\right] ; \phi_{a o}$ is the value of $\phi_{a}$ under the reference pressure $P_{0} ; c$ is the total compressibility of reservoir, $\left[\left(\mathrm{LT}^{2}\right) / \mathrm{M}\right] ; t$ is the transfer flow time, [T].

Initial and boundary conditions are given by:

$$
\begin{gathered}
P=P_{0}, \quad-L / 2 \leq x \leq L / 2, \quad t=0 \\
P=P_{f}, \quad x= \pm L / 2, \quad t>0 .
\end{gathered}
$$

Using separation variable method and Bessel function and combining equation (6) with equation (7) (see Appendix A for details):

$\frac{\bar{P}-P_{0}}{P_{f}-P_{o}}=1-\sum_{n=0}^{\infty} \frac{(-1)^{n} 8 \times \mathrm{e}^{(2 n+1)^{2}}}{L \pi^{2}(2 n+1)^{2}} \delta \exp \left(-\frac{1}{M} \frac{2^{2 D_{T}-2} \pi^{2} D_{T}^{2}}{L^{2 D_{T}}} t\right)$,

where $M=\frac{\phi_{a o} \mu c}{K_{a} \lambda_{\max }^{2\left(D_{T}-1\right)}}$ is a constant.

The partial derivative of equation (8) with respect to time $t$ gives:

$$
\begin{aligned}
\frac{\partial \bar{P}}{\partial t} \frac{1}{P_{f}-P_{0}} & =\frac{1}{M} \frac{2^{2 D_{T}-2} \pi^{2} D_{T}^{2}}{L^{2 D_{T}}} \\
& \times \sum_{n=0}^{\infty} \frac{(-1)^{n} 8 \times \mathrm{e}^{(2 n+1)^{2}}}{L \pi^{2}(2 n+1)^{2}} \delta \exp \left(-\frac{1}{M} \frac{2^{2 D_{T}-2} \pi^{2} D_{T}^{2}}{L^{2 D_{T}}} t\right) \\
& =\frac{1}{M} \frac{2^{2 D_{T}-2} \pi^{2} D_{T}^{2}}{L^{2 D_{T}}}\left(1-\frac{\bar{P}-P_{0}}{P_{f}-P_{0}}\right) .
\end{aligned}
$$


Equation (9) can be also written as:

$$
\frac{\partial \bar{P}}{\partial t}=-\frac{K_{a} \lambda_{\max }^{2\left(D_{T}-1\right)}}{\phi_{a o} \mu c} \frac{2^{2 D_{T}-2} \pi^{2} D_{T}^{2}}{L^{2 D_{T}}}\left(\bar{P}-P_{f}\right) .
$$

The 1D transfer function can be expressed as:

$$
q=-\frac{\partial(\rho \phi)_{m}}{\partial t}=-\rho \phi_{a o} c \frac{\partial \bar{P}}{\partial t}\left(\frac{\lambda_{\max }}{L}\right)^{1-D_{T}} .
$$

Substituting equation (10) into equation (11) yields:

$$
q=\frac{\rho}{\mu} K_{a} \frac{2^{2 D_{T}-2} \pi^{2} D_{T}^{2}}{L^{1+D_{T}}} \lambda_{\max }^{D_{T}-1}\left(\bar{P}-P_{f}\right) .
$$

Compared to the transfer function (Eq. (1)), shape factor in $1 \mathrm{D}$ flow can be expressed as:

$$
\sigma=\pi^{2} \frac{2^{2 D_{T}-2} D_{T}^{2}}{L^{1+D_{T}}} \lambda_{\max }^{D_{T}-1}
$$

From equation (13), the proposed shape factor considers the effect of flow channel bending and micro pore structure of matrix. Specifically, shape factor is clearly related to tortuosity fractal dimension, maximum pore diameter and characteristic length of matrix.

\subsection{Transfer function and shape factor of 2D flow}

In 2D model, the matrix is surrounded by four fractures, with two groups of parallel fractures. Fracture space is $L$. For the 2D matrix-fracture system, the pressure diffusion behavior from matrix to fracture can be similar to a circular region (Fig. 4). The equivalent radius $R$ of matrix is:

$$
L^{2}=\pi R^{2} \Rightarrow R=\frac{L}{\sqrt{\pi}} .
$$

The 2D matrix fractal pressure diffusion equation can be given by (Kong et al., 2007):

$$
\frac{\partial^{2} P}{\partial r^{2}}+\frac{2-D_{T}}{r} \frac{\partial P}{\partial r}=\frac{\phi_{a o} \mu c}{K_{a}}\left(\frac{r}{\lambda_{\max }}\right)^{2\left(D_{T}-1\right)} \frac{\partial P}{\partial t},
$$

where $r$ is the distance, [L].

Initial and boundary conditions are given by:

$$
\begin{gathered}
P=P_{0}, \quad 0 \leq r \leq R, \quad t=0 \\
P=P_{f}, \quad r=R, \quad t>0 .
\end{gathered}
$$

Using separation variable method, the analytical solution of equation (15) can be obtained in the form of Bessel function (see Appendix B for details):

$$
\begin{aligned}
P= & P_{f}+r^{\frac{D_{T}-1}{2}} \\
& \times \sum_{m=1}^{\infty} 2\left(P_{0}-P_{f}\right) \eta \exp \left[-\frac{1}{M}\left(\frac{D_{T} I_{1}}{R^{D_{T}}}\right)^{2} t\right],
\end{aligned}
$$

where $\quad \eta=\sum_{m=1}^{\infty} \frac{1}{J_{v-1}\left(I_{1}\right)}\left[-D_{T}^{\frac{1-D_{T}}{2 D_{T}}}\left(\frac{R^{D_{T}}}{D_{T}}\right)^{\frac{1-3 D_{T}}{2 D_{T}}}\left(\frac{D_{T} I_{1}}{R^{D} T}\right)^{-\frac{3 D_{T}+1}{2 D_{T}}}\right]$ $\times J_{v}\left(\frac{r^{D_{T} I_{1}}}{R^{D_{T}}}\right), J_{v}(x)$ is the Bessel function, $v$ is the order of Bessel function, $I_{1}$ is the positive zero point of Bessel function (the solution code of $I_{1}$ is shown in Appendix C), it depends on the value of $v$, and $v=\frac{D_{T}-1}{2 D_{T}}$.

Assuming that the area of matrix $A_{a v}$ is equal to $\pi r^{2}$. The average pressure of matrix is as follows:

$$
\bar{P}=\frac{1}{\pi R^{2}} \int_{0}^{R} P \mathrm{~d} A_{a v} .
$$

Substituting equation (17) into equation (18) yields:

$$
\begin{aligned}
\bar{P}= & P_{f}+\frac{4}{R^{2}} \sum_{m=1}^{\infty}\left(P_{f}-P_{0}\right) \int_{0}^{R} r^{\frac{D_{T}+1}{2}} \eta \mathrm{d} r \\
& \times \exp \left[-\frac{1}{M}\left(\frac{D_{T} I_{1}}{R^{D_{T}}}\right)^{2} t\right] .
\end{aligned}
$$

Both sides of equation (19) are subtracted by $P_{0}$ and then divided by $P_{f}-P_{0}$. Thus, equation (19) can be expressed as:

$$
\begin{aligned}
\frac{\bar{P}-P_{0}}{P_{f}-P_{0}}= & 1+\frac{4}{R^{2}} \sum_{m=1}^{\infty} \int_{0}^{R} r^{\frac{D_{T}+1}{2}} \eta \mathrm{d} r \\
& \times \exp \left[-\frac{1}{M}\left(\frac{D_{T} I_{1}}{R^{D_{T}}}\right)^{2} t\right] .
\end{aligned}
$$

The partial derivative of equation (20) with respect to $t$ can be expressed as:

$$
\begin{aligned}
\frac{\partial \bar{P}}{\partial t} \frac{1}{P_{f}-P_{0}}= & -\frac{1}{M}\left(\frac{D_{T} I_{1}}{R^{D_{T}}}\right)^{2} \frac{4}{R^{2}} \sum_{m=1}^{\infty} \int_{0}^{R} r^{\frac{D_{T}+1}{2}} \eta \mathrm{d} r \\
& \times \exp \left[-\frac{1}{M}\left(\frac{D_{T} I_{1}}{R^{D_{T}}}\right)^{2} t\right] \\
= & -\frac{1}{M}\left(\frac{D_{T} I_{1}}{R^{D_{T}}}\right)^{2}\left(\frac{\bar{P}-P_{0}}{P_{f}-P_{0}}-1\right) .
\end{aligned}
$$

Equation (21) also can be written as:

$$
\frac{\partial \bar{P}}{\partial t}=-\frac{K_{a} \lambda_{\max }^{2\left(D_{T}-1\right)}}{\phi_{a o} \mu c}\left(\frac{D_{T} I_{1}}{R^{D_{T}}}\right)^{2}\left(\bar{P}-P_{f}\right) .
$$

The 2D transfer function can be expressed as:

$$
q=-\frac{\partial(\rho \phi)_{m}}{\partial t}=-\rho \phi_{a o} c \frac{\partial \bar{P}}{\partial t}\left(\frac{\lambda_{\max }}{R}\right)^{1-D_{T}}
$$

Substituting equation (22) into equation (23) yields:

$$
q=\frac{\rho}{\mu} K_{a} \frac{D_{T}^{2} I_{1}^{2}}{R^{1+D_{T}}} \lambda_{\max }^{D_{T}-1}\left(\bar{P}-P_{f}\right) .
$$




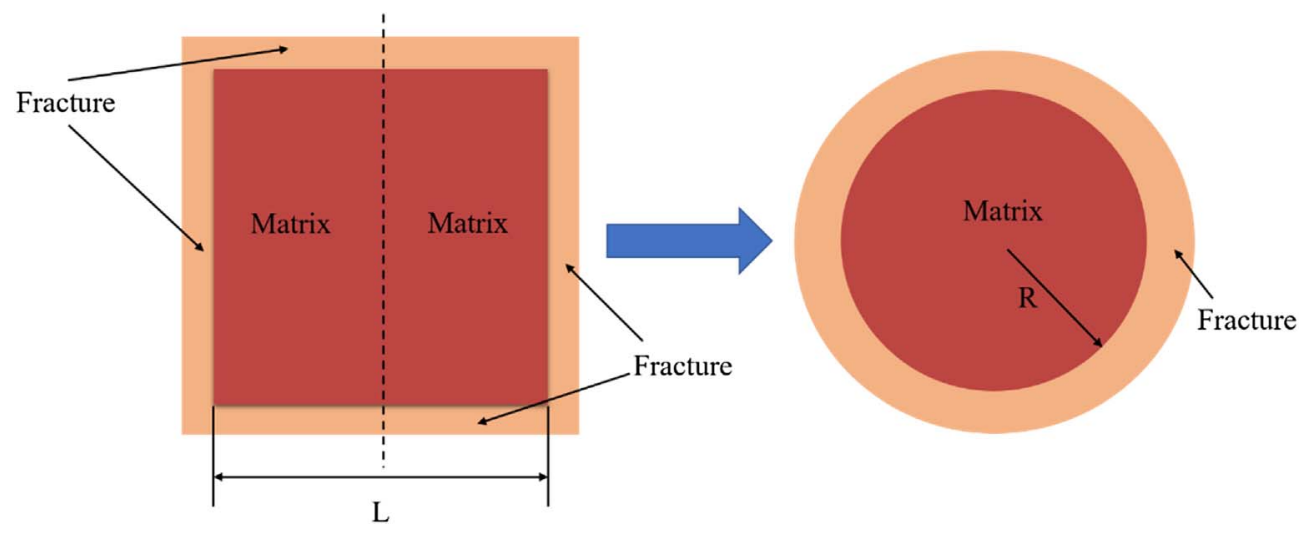

Fig. 4. Schematic of $2 \mathrm{D}$ matrix-fracture system.

Consequently, the 2D shape factor can be expressed as:

$$
\sigma=\frac{D_{T}^{2} I_{1}^{2}}{R^{1+D_{T}}} \lambda_{\max }^{D_{T}-1}
$$

Substituting equation (14) into equation (25) yields:

$$
\sigma=\pi^{\frac{1+D_{T}}{2}} \frac{D_{T}^{2} I_{1}^{2}}{L^{1+D_{T}}} \lambda_{\max }^{D_{T}-1} .
$$

The presented 2D shape factor also considers the effect of tortuosity fractal dimension, maximum pore diameter and characteristic length of matrix. Moreover, the value of the positive zero point of Bessel function is related to the value of tortuosity fractal dimension.

\subsection{Transfer function and shape factor of 3D flow}

In 3D model, the matrix is surrounded by six fractures, with three groups of parallel fractures, which have a spacing $L$. For the 3D matrix-fracture system, the pressure diffusion behavior from matrix to fracture can be approximately regarded as a sphere (Fig. 5). The equivalent radius $R$ of matrix is:

$$
L^{3}=\frac{4}{3} \pi R^{3} \Rightarrow R=L^{3} \sqrt{\frac{0.75}{\pi}} .
$$

The 3D matrix fractal pressure diffusion equation can be given by (Kong et al., 2007):

$$
\frac{\partial^{2} P}{\partial r^{2}}+\frac{3-D_{T}}{r} \frac{\partial P}{\partial r}=\frac{\phi_{a o} \mu c}{K_{a}}\left(\frac{r}{\lambda_{\max }}\right)^{2\left(D_{T}-1\right)} \frac{\partial P}{\partial t} .
$$

Initial and boundary conditions are given by:

$$
\begin{gathered}
P=P_{0}, \quad 0 \leq r \leq R, \quad t=0 \\
P=P_{f}, \quad r=R, \quad t>0 .
\end{gathered}
$$

Using separation variable method, the analytical solution of equation (28) can be obtained in the form of Bessel function (see Appendix B for details):

$$
P=P_{f}+r^{\frac{D_{T}-2}{2}} \sum_{m=1}^{\infty} 2\left(P_{f}-P_{0}\right) \varpi \exp \left[-\frac{1}{M}\left(\frac{D_{T} I_{2}}{R^{D_{T}}}\right)^{2} t\right],
$$

where $\bar{\omega}=\frac{1}{J_{v-1}\left(I_{2}\right)} D_{T}^{-\frac{\left|D_{T}-2\right|}{2 D_{T}}}\left(\frac{R^{D_{T}}}{D_{T}}\right)^{-\frac{\left|D_{T}-2\right|+2 D_{T}}{2 D_{T}}}\left(\frac{D_{T} I_{2}}{R^{D} T}\right)^{\frac{\left|D_{T}-2\right|-4 D_{T}}{2 D_{T}}}$ $\times J_{v}\left(\frac{r^{D_{T} I_{2}}}{R^{D_{T}}}\right), I_{2}$ is the positive zero point of Bessel function (the solution code of $I_{2}$ is shown in Appendix C), it depends on the value of $v$, and $v=\frac{\left|D_{T}-2\right|}{2 D_{T}}$.

Assuming that the volume of matrix $V_{a v}$ is equal to $\frac{4}{3} \pi r^{3}$, the average pressure of matrix is as follows:

$$
\bar{P}=\frac{1}{\frac{4}{3} \pi R^{3}} \int_{0}^{R} P \mathrm{~d} V_{a v}
$$

Substituting equation (30) into equation (31) yields:

$$
\begin{aligned}
\bar{P}= & P_{f}+\frac{6}{R^{3}} \sum_{m=1}^{\infty}\left(P_{f}-P_{0}\right) \int_{0}^{R} r^{\frac{D_{T+2}}{2}} \varpi \mathrm{d} r \\
& \times \exp \left[-\frac{1}{M}\left(\frac{D_{T} I_{2}}{R^{D_{T}}}\right)^{2} t\right] .
\end{aligned}
$$

Both sides of equation (32) are subtracted by $P_{0}$ and then divided by $P_{f}-P_{0}$. Thus, equation (32) can be expressed as:

$$
\frac{\bar{P}-P_{0}}{P_{f}-P_{o}}=1+\frac{6}{R^{3}} \sum_{m=1}^{\infty} \int_{0}^{R} r^{\frac{D_{T+2}}{2}} \operatorname{\varpi d} r \exp \left[-\frac{1}{M}\left(\frac{D_{T} I_{2}}{R^{D_{T}}}\right)^{2} t\right] .
$$

The partial derivative of equation (33) with respect to $t$ can be expressed as: 

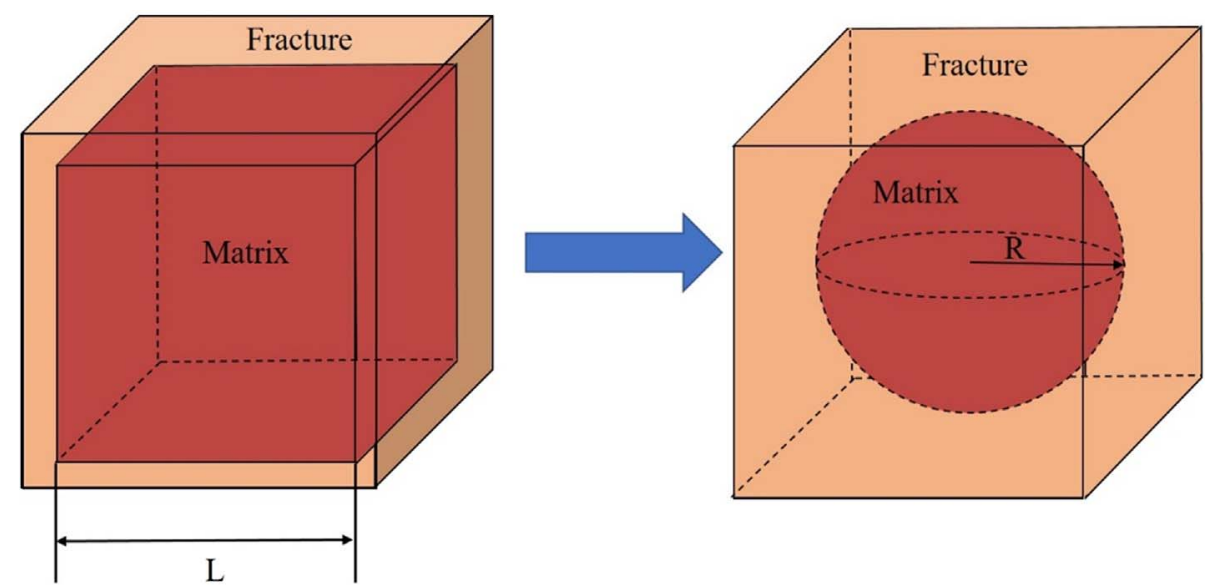

Fig. 5. Schematic of 3D matrix-fracture system.

$$
\begin{aligned}
\frac{\partial \bar{P}}{\partial t} \frac{1}{P_{f}-P_{0}}= & -\frac{1}{M}\left(\frac{D_{T} I_{2}}{R^{D_{T}}}\right)^{2} \frac{6}{R^{3}} \sum_{m=1}^{\infty} \int_{0}^{R} r^{\frac{D_{T}+2}{2}} \varpi \mathrm{d} r \\
& \times \exp \left[-\frac{1}{M}\left(\frac{D_{T} I_{2}}{R^{D_{T}}}\right)^{2} t\right] \\
= & -\frac{1}{M}\left(\frac{D_{T} I_{2}}{R^{D_{T}}}\right)^{2}\left(\frac{\bar{P}-P_{0}}{P_{f}-P_{0}}-1\right) .
\end{aligned}
$$

Equation (34) can be also written as:

$$
\frac{\partial \bar{P}}{\partial t}=-\frac{K_{a} \lambda_{\max }^{2\left(D_{T}-1\right)}}{\phi_{a o} \mu c}\left(\frac{D_{T} I_{2}}{R^{D_{T}}}\right)^{2}\left(\bar{P}-P_{f}\right)
$$

The 3D transfer function can be expressed as:

$$
q=-\frac{\partial(\rho \phi)_{m}}{\partial t}=-\rho \phi_{a o} c \frac{\partial \bar{P}}{\partial t}\left(\frac{\lambda_{\max }}{R}\right)^{1-D_{T}} .
$$

Substituting equation (35) into equation (36) yields:

$$
q=\frac{\rho}{\mu} K_{a} \frac{D_{T}^{2} I_{2}^{2}}{R^{1+D_{T}}} \lambda_{\max }^{D_{T}-1}\left(\bar{P}-P_{f}\right) .
$$

Consequently, the 3D shape factor can be expressed as:

$$
\sigma=\frac{D_{T}^{2} I_{2}^{2}}{R^{1+D_{T}}} \lambda_{\max }^{D_{T}-1} .
$$

Substituting equation (27) into equation (38) gives:

$$
\sigma=\left(\frac{4}{3} \pi\right)^{\frac{1+D_{T}}{3}} \frac{D_{T}^{2} I_{2}^{2}}{L^{1+D_{T}}} \lambda_{\max }^{D_{T}-1}
$$

Compared with those shape factors proposed by predecessors, the fractal shape factor accounts for the influences of two additional parameters: tortuosity fractal dimension and maximum pore diameter of matrix. The effect of microstructure on shape factor directly affects fluids transfer in matrix-fracture system. When the flow channels in matrix are approximately straight, tortuosity fractal dimension of matrix $D_{T}=1$, our shape factor model has the

Table 1. The shape factors defined by various models.

\begin{tabular}{llll}
\hline Researcher(s) & \multicolumn{1}{c}{$1 \mathrm{D}$} & \multicolumn{1}{c}{$2 \mathrm{D}$} & \multicolumn{1}{c}{$3 \mathrm{D}$} \\
\hline Warren and Root $(1963)$ & $12 / L^{2}$ & $32 / L^{2}$ & $60 / L^{2}$ \\
Kazemi et al. $(1976)$ & $4 / L^{2}$ & $8 / L^{2}$ & $12 / L^{2}$ \\
Lim and Aziz $(1995)$ & $\pi^{2} / L^{2}$ & $18.17 / L^{2}$ & $25.67 / L^{2}$ \\
This paper $\left(D_{T}=1.1\right.$, & $11.35 / L^{2.1}$ & $20.38 / L^{2.1}$ & $24.77 / L^{2.1}$ \\
$\left.\lambda_{\max }=0.15 \mathrm{~mm}\right)$ & & & \\
\hline
\end{tabular}

similar value with Lim and Aziz (1995) model. When the flow channels in matrix are curved, according to the experimental observation, Yin et al. (2017) presented that tortuosity fractal dimension of sandstone, coal and shale media is generally ranging from 1.1 to 2.3. Nelson (2009) reported that pore diameters are usually greater than $2 \mu \mathrm{m}$ in conventional reservoirs, ranging from 2 to $0.03 \mu \mathrm{m}$ and from 0.1 to $0.005 \mu \mathrm{m}$ in tight-gas sandstone and shale, respectively. Within the reasonable value range $D_{T}=1.1$ and $\lambda_{\max }=0.15 \mathrm{~mm}$ to calculate the positive zero point of Bessel function, we can get $I_{1}=2.474$ and $I_{2}=3.013$, and shape factors are $11.35 / L^{2.1}, 20.38 / L^{2.1}$ and $24.77 / L^{2.1}$ for $1 \mathrm{D}, 2 \mathrm{D}$ and $3 \mathrm{D}$ spaces, respectively. The shape factors predicted by Warren and Root (1963), Kazemi et al. (1976), Lim and Aziz (1995) and our model are presented in Table 1.

\section{Model verification}

Here, the commercial reservoir simulator ECLIPSE is applied instead of the laboratory experiment to verify the proposed fractal shape factor. Three types of single-porosity fine-grid model are developed using ECLIPSE, and offer reference curve for the dual-porosity models with different shape factors. In the single-porosity fine-grid models, matrix is discretized that grids near fractures are finer than others, and fracture spacing is $10 \mathrm{~cm}$ (Fig. 6). The dual-porosity models only have one cubic grid with a size of $50 \mathrm{~cm}$, which 

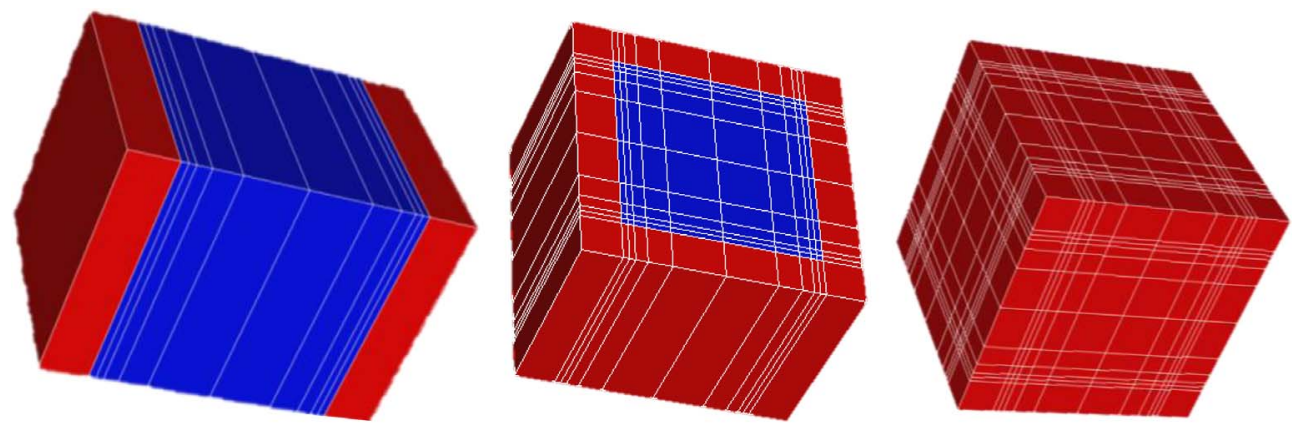

SATNum

Matrix

(a) (b)
Fracture

(c)

Fig. 6. Schematic of single-porosity fine-grid models for (a) 1D, (b) 2D, and (c) 3D flow.

is mainly controlled by shape factor in transfer function, and fracture spacing is $50 \mathrm{~cm}$. The basic parameters used in these models are shown in Table 2.

Curves of cumulative production over time under 1D, $2 \mathrm{D}$ and $3 \mathrm{D}$ flows are displayed in Figures $7 \mathrm{a}-7 \mathrm{c}$, respectively. The results of single-porosity fine-grid models are taken as the reference curves. Results show that the fractal shape factors have relatively accurate matching degree with the reference curves. Clearly, the curvature of the fluid channels can't be ignored in the real rocks. Both tortuosity fractal dimension and maximum pore diameter have an important role in the fluid flow in porous media (Yun et al., 2009; Ye et al., 2019; Huang et al., 2020). Thus, there will be a great error in the fluid exchange term if neglecting the effects of tortuosity fractal dimension and maximum pore diameter of matrix on the shape factor. The proposed

Table 2. Basic parameters used in numerical simulation.

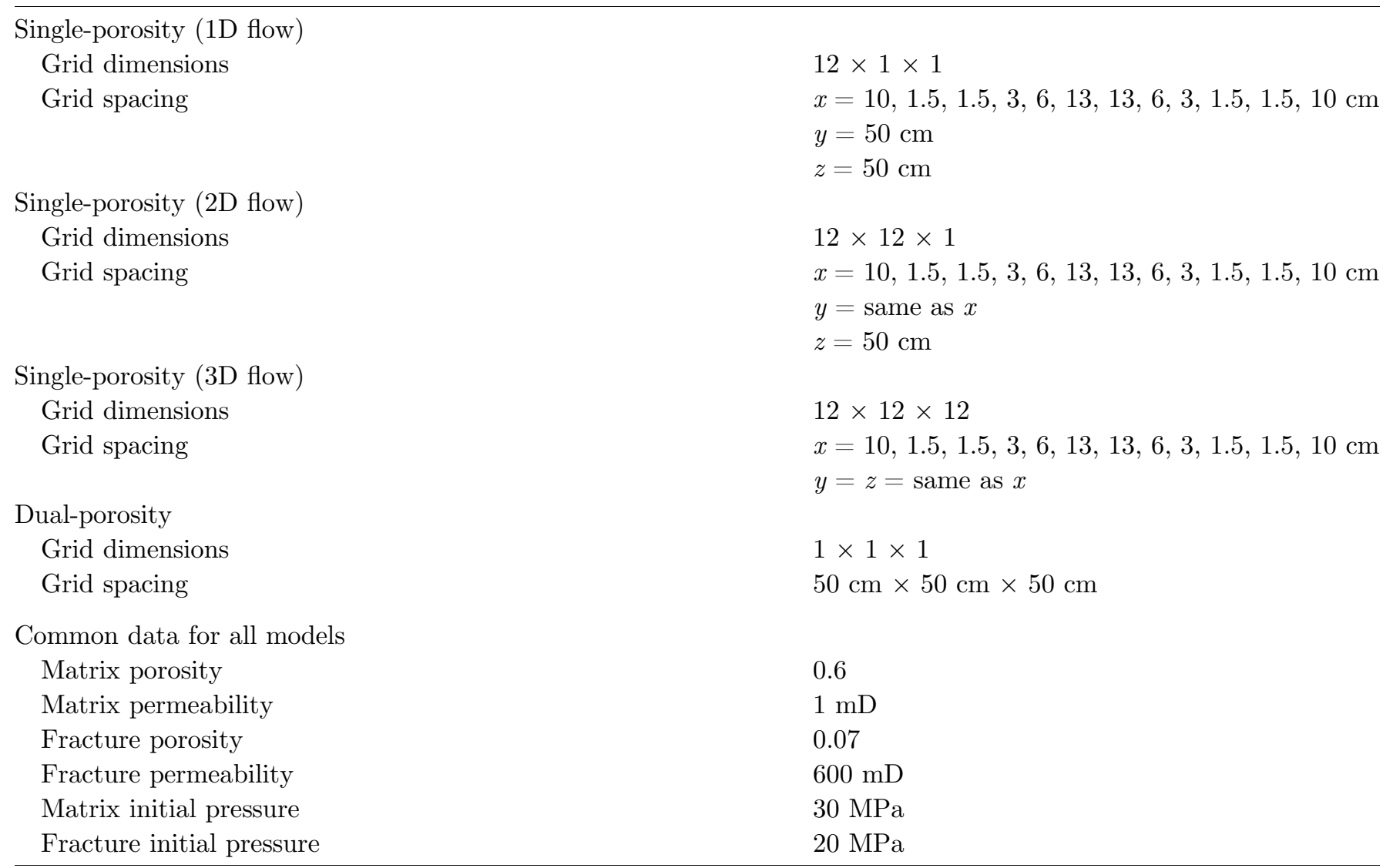



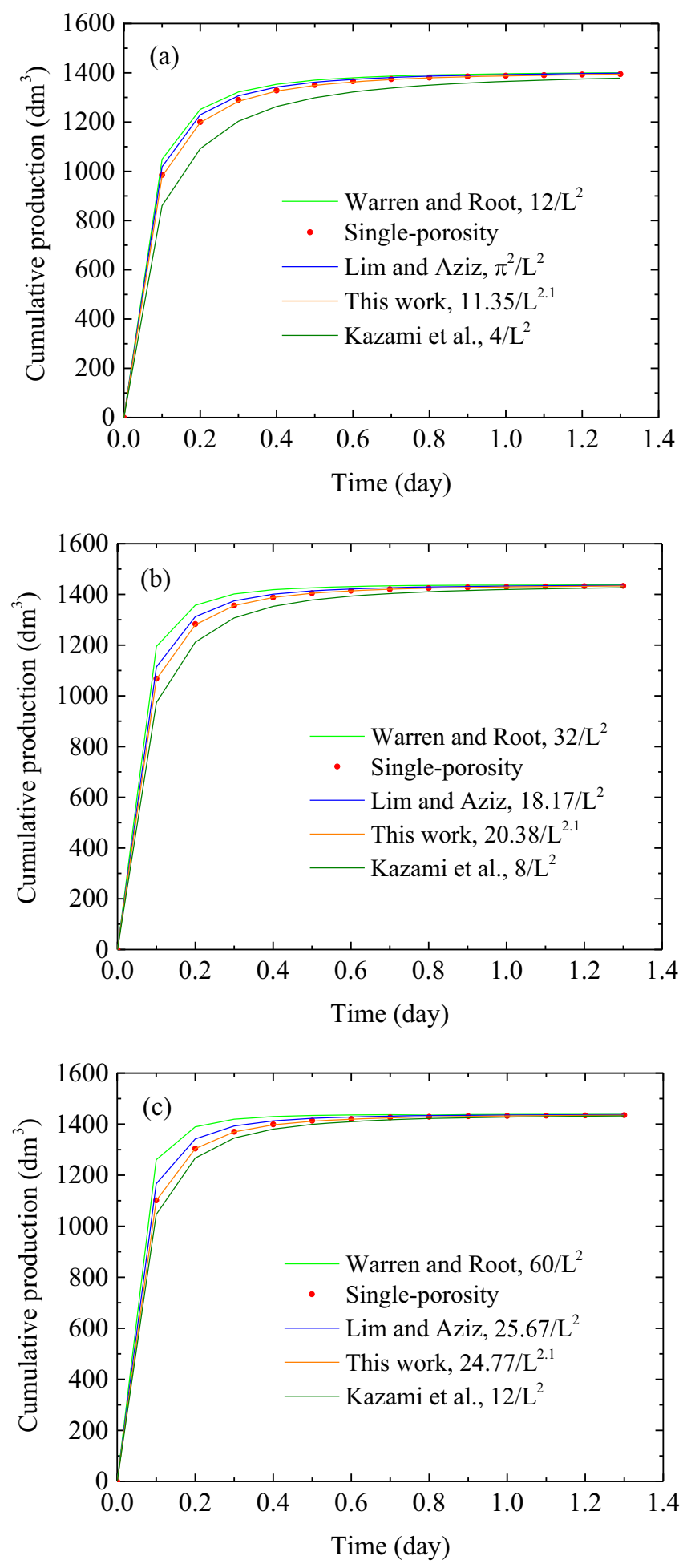

Fig. 7. Cumulative production curves of dual-porosity model with different shape factors and single-porosity fine-grid model, (a) $1 \mathrm{D}$ flow; (b) 2D flow; (c) 3D flow.

fractal shape factor can be used to explain the impacts of tortuosity fractal dimension and maximum pore diameter on the fluid exchange rate, and give a better prediction of production rates and recoveries.
Table 3. The positive zero point of Bessel function at different values of tortuosity fractal dimension.

\begin{tabular}{lcc}
\hline Tortuosity fractal dimension & $I_{1}$ & $I_{2}$ \\
\hline 1.0 & 2.405 & 3.142 \\
1.1 & 2.474 & 3.013 \\
1.2 & 2.532 & 2.902 \\
1.3 & 2.580 & 2.809 \\
1.4 & 2.622 & 2.728 \\
1.5 & 2.658 & 2.658 \\
\hline
\end{tabular}

\section{Results and discussion}

The fractal shape factor model presented in this paper (Eqs. (13), (26) and (39)) is in term of tortuosity fractal dimension, maximum pore diameter and characteristic length of matrix. The impacts of parameters on shape factor are studied in this part to understand the sensitivity to shape factor. It is generally believed that the permeability of

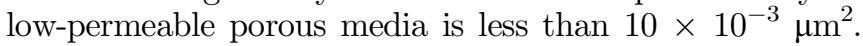
With the decrease of permeability, the characteristics of nonlinear flow become obvious. Since the derivation of the presented fractal shape factor model follows Darcy's law, so according to the experimental data (Yin et al., 2017), tortuosity fractal dimension of matrix is roughly less than 1.5. Six values of tortuosity fractal dimension of matrix are selected as 1.0, 1.1, 1.2, 1.3, 1.4 and 1.5, respectively, which reflect the different curvature of flow channels in porous media. A range of maximum pore diameters of matrix that varies from $0.1 \mu \mathrm{m}$ to $2 \mathrm{~mm}$, covering conventional rocks, tight sandstone and shale. The fractal shape factor model contains the positive zero point of Bessel function, which is related to tortuosity fractal dimension. So, we first solve the positive zero point of the Bessel function at different values of tortuosity fractal dimension, as shown in Table 3.

The values of the fractal shape factor decreases as tortuosity fractal dimension increases (Fig. 8). Since the larger tortuosity fractal dimension is, the more curved the flow channel is, which will lead to greater flow resistance, thus reducing the fluid exchange mass between matrix and fracture. However, when tortuosity fractal dimension is larger than 1.25 , the shape factor of $2 \mathrm{D}$ flow is greater than that of $3 \mathrm{D}$ flow. Since the number of fractures around matrix increases, a larger flow cross-sectional area is formed, resulting in a greater fluid exchange rate between matrix and fracture. The shape factor of $2 \mathrm{D}$ flow should be less than that of $3 \mathrm{D}$ flow. Figure 8 indicates the presented fractal shape factor model is suitable for tortuosity fractal dimension less than 1.25.

The fractal shape factor growths with the increase of maximum pore diameter of matrix (Fig. 9). The presented fractal shape factor model is applicable in all range of maximum pore diameter of matrix, indicating that the presented fractal shape factor model is suitable for dualporosity media at different scales and the presented fractal shape factor varies at different scales.

The fractal shape factor decreases with the increase of characteristic length of matrix (Fig. 10). The increase of 


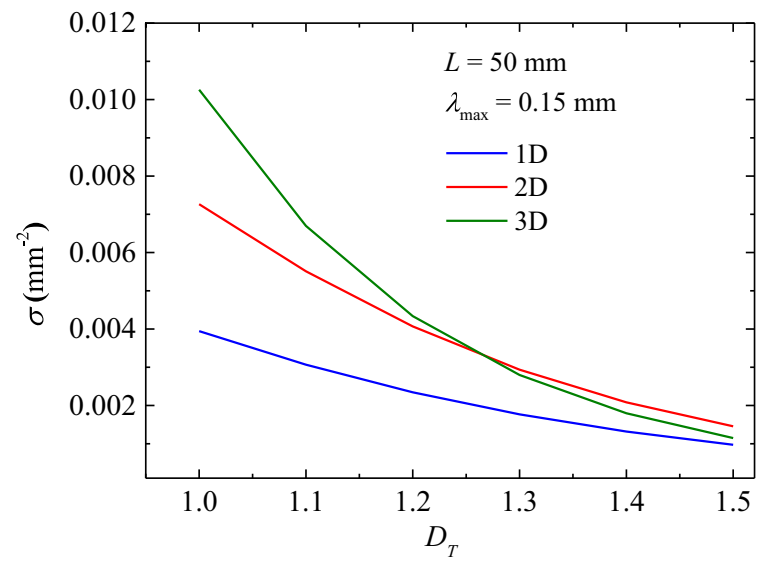

Fig. 8. The impact of tortuosity fractal dimension of matrix on the fractal shape factor.

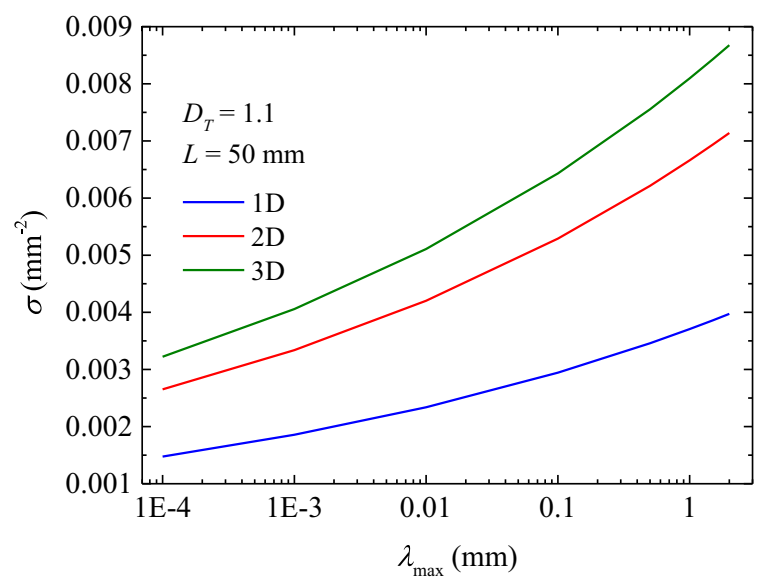

Fig. 9. The impact of maximum pore diameter of matrix on the fractal shape factor.

characteristic length of matrix means that the fluid flow time is longer, reducing the rate of fluids transfer between matrix and fracture.

In order to further understand the fluids transfer mechanism between matrix and fracture, different shape factor models have been presented in the literature. Warren and Root (1963) combined an integral material balance to derive shape factors are $12 / L^{2}, 32 / L^{2}$ and $60 / L^{2}$ for $1 \mathrm{D}$, 2D and 3D, respectively. Moreover, Lim and Aziz (1995) used approximating analytical solution of matrix pressure diffusion equation to derived shape factors are $\pi^{2} / L^{2}$, $18.17 / L^{2}$ (or $2 \pi^{2} / L^{2}$ ) and $25.67 / L^{2}$ (or $3 \pi^{2} / L^{2}$ ) for $1 \mathrm{D}$, 2D and 3D, respectively. He et al. (2017) considered the influence of tortuosity $(\tau)$ and introduced the modified shape factors are $\pi^{2} /\left(\tau L^{2}\right), 18 /\left(\tau L^{2}\right)$ and $26 /\left(\tau L^{2}\right)$ for $1 \mathrm{D}, 2 \mathrm{D}$ and $3 \mathrm{D}$, respectively. When these shape factors are applied in the transfer function to investigate the fluids transfer between matrix and fracture, the impact of matrix microscopic pore structure is usually neglected, which is deviated from the actual situation. The presented fractal shape factor considers the impacts of tortuosity fractal

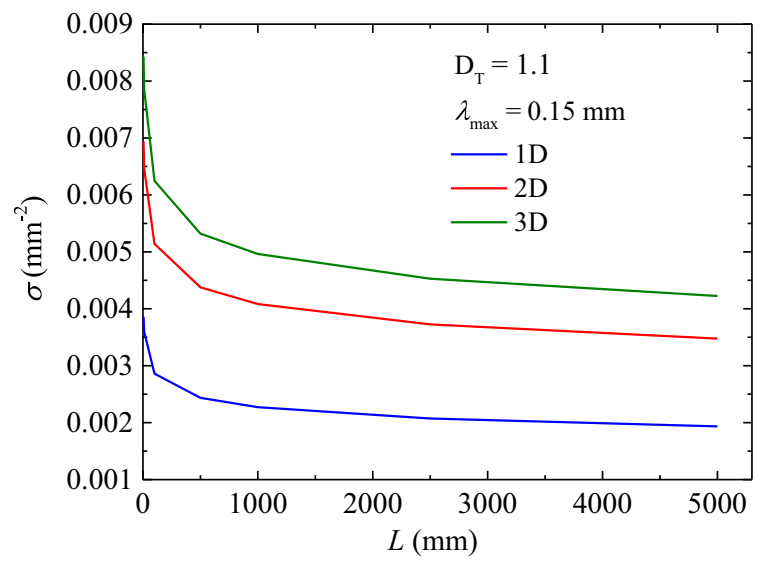

Fig. 10. The impact of characteristic length of matrix on the fractal shape factor.

dimension, maximum pore diameter and characteristic length of matrix. Compared with the previous models, the proposed fractal shape factor model can more accurately characterize the impact of microscopic pore structure on the rate of fluids transfer between matrix and fracture in fractured reservoirs.

\section{Conclusion}

We established new transfer function and shape factor to investigate the fluids transfer between matrix and fracture in fractured reservoirs. The analytical expression for fractal shape factors in $1 \mathrm{D}, 2 \mathrm{D}$ and $3 \mathrm{D}$ spaces are derived via the fractal pressure diffusion equation. The presented fractal shape factor model is authenticated by comparing dualporosity model with single-porosity fine-grid model. Furtherly, sensitivity analysis of model parameters is carried out to understand their effects on fractal shape factor. Some conclusions can be obtained:

1. For different porous media, the fractal shape factor can provide corresponding values. Applying the fractal shape factor to transfer function will help us better understand the impact of microscopic pore structure on fluid exchange law between matrix and fracture.

2. There is a positive correlation between the fractal shape factor and maximum pore diameter of matrix. The fractal shape factor decreases with the growth of tortuosity fractal dimension and characteristic length of matrix.

3. When tortuosity fractal dimension is roughly less than 1.25 , the fractal shape factor has practical application value in different scales.

Acknowledgments. This work is supported by the Strategic Priority Research Program of the Chinese Academy of Sciences (No. XDA14010302), the National Natural Science Foundation of China (No. 51904279), and the Fundamental Research Funds for the Central Universities (China University of Geosciences, Wuhan) (No. CUGGC04). 


\section{References}

Acuna J.A., Ershaghi I., Yortsos Y.C. (1995) Practical application of fractal pressure transient analysis of naturally fractured reservoirs, SPE Form. Eval. 10, 3, 173-179.

Adler P.M. (1996) Transports in fractal porous media, J. Hydrol. 187, 195-213.

Barenblatt G.I., Zheltov I.P., Kochina I.N. (1960) Basic concepts in the theory of seepage of homogeneous liquids in fissured rocks, J. Appl. Math. Mech. 24, 5, 1286-1303.

Behnoudfar P., Asadi M.B., Gholilou A., Zendehboudi S. (2019) A new model to conduct hydraulic fracture design in coalbed methane reservoirs by incorporating stress variations, J. Pet. Sci. Eng. 174, 1208-1222.

Cao R., Xu Z., Cheng L., Peng Y., Wang Y., Guo Z. (2019) Study of single phase mass transfer between matrix and fracture in tight oil reservoirs, Geofluids 2019, 1-11.

Chang J., Yortsos Y.C. (1990) Pressure-transient analysis of fractal reservoirs, SPE Form. Eval. 5, 1, 31-38.

Coats K.H. (1989) Implicit compositional simulation of singleporosity and dual-porosity reservoirs, in: SPE Symposium on Reservoir Simulation, Houston, Texas, 6-8 February, Society of Petroleum Engineers.

Costa A. (2006) Permeability-porosity relationship: A reexamination of the Kozeny-Carman equation based on a fractal pore-space geometry assumption, Geophys. Res. Lett. 33, 2, L02318.

Erol S., Fowler S.J., Harcouët-Menou V., Laenen B. (2017) An analytical model of porosity-permeability for porous and fractured media, Transp. Porous Media 120, 2, 327-358.

Fan D., Ettehadtavakkol A. (2017) Semi-analytical modeling of shale gas flow through fractal induced fracture networks with microseismic data, Fuel 193, 444-459.

Flamenco-Lopez F., Camacho-Velazquez R. (2001) Fractal transient pressure behavior of naturally fractured reservoirs, in: SPE Annual Technical Conference and Exhibition, 30 September-3 October, New Orleans, Louisiana, Society of Petroleum Engineers.

Hassanzadeh H., Pooladidarvish M. (2006) Effects of fracture boundary conditions on matrix-fracture transfer shape factor, Transp. Porous Media 64, 1, 51-71.

He Y., Chen X., Zhang Y., Yu W. (2017) Modeling interporosity flow functions and shape factors in low-permeability naturally fractured reservoir, J. Pet. Sci. Eng. 156, 110-117.

Huang T., Du P., Peng X., Wang P., Zou G. (2020) Pressure drop and fractal non-Darcy coefficient model for fluid flow through porous media, J. Pet. Sci. Eng. 184, 106579.

Katz A.J., Thompson A.H. (1985) Fractal sandstone pores: Implications for conductivity and pore formation, Phys. Rev. Lett. 5412, 1325-1328.

Kazemi H., Merrill L.S., Porterfield K.L., Zeman P.R. (1976) Numerical simulation of water-oil flow in naturally fractured reservoirs, SPE J. 16, 6, 317-326.

Kong X., Li D., Lu D. (2007) Basic formulas of fractal seepage and type-curves of fractal reservoirs, Journal of Xi'an Shiyou University (Natural Science Edition) 22, 2, 1-10.

Kong X., Li D., Lu D. (2009) Transient pressure analysis in porous and fractured fractal reservoirs, Sci. China, Ser. E: Technol. Sci. 52, 9, 2700-2708.

Krohn C.E. (1988) Fractal measurements of sandstones, shales, and carbonates, J. Geophys. Res. 93, 3297-3305.

Li Q., Xing H., Liu J., Liu X. (2015) A review on hydraulic fracturing of unconventional reservoir, Petroleum 1, 1, 8-15.
Lian P.Q., Duan T.Z., Xu R., Li L.L., Li M. (2018) Pressure behavior of shale-gas flow in dual porous medium based on fractal theory, Interpretation 6, 4, SN1-SN10.

Lim K.T., Aziz K. (1995) Matrix-fracture transfer shape factors for dual-porosity simulators, J. Pet. Sci. Eng. 13, 3, 169-178.

Nelson P.H. (2009) Pore-throat sizes in sandstones, tight sandstones, and shales, AAPG Bull. 93, 3, 329-340.

Noetinger B., Estebenet T. (2000) Up-scaling of double porosity fractured media using continuous-time random walks methods, Transp. Porous Media 39, 3, 315-337.

Ranjbar E., Hassanzadeh H., Chen Z. (2011) Effect of fracture pressure depletion regimes on the dual-porosity shape factor for flow of compressible fluids in fractured porous media, $A d v$. Water Resour. 34, 12, 1681-1693.

Saboorian-Jooybari H., Ashoori S., Mowazi G. (2015) A new transient matrix/fracture shape factor for capillary and gravity imbibition in fractured reservoirs, Energy Sources Part A 37, 23, 2497-2506.

Sarma P., Aziz K. (2004) New transfer functions for simulation of naturally fractured reservoirs with dual porosity models, in: SPE Annual Technical Conference and Exhibition, Houston, Texas, 26-29 September, Society of Petroleum Engineers.

Thomas L.K., Dixon T.N., Pierson R.G. (1983) Fractured reservoir simulation, SPE J. 23, 1, 42-54.

Ueda Y., Murata S., Watanabe Y., Funatsu K. (1989) Investigation of the shape factor used in the dual-porosity reservoir simulator, in: SPE Asia-Pacific Conference, 13-15 September, Sydney, Society of Petroleum Engineers.

Vishkai M., Gates I. (2019) On multistage hydraulic fracturing in tight gas reservoirs: Montney Formation, Alberta, Canada, J. Pet. Sci. Eng. 174, 1127-1141.

Wang W., Yuan B., Su Y., Sheng G., Yao W., Gao H., Wang K. (2018) A composite dual-porosity fractal model for channelfractured horizontal wells, Eng. Appl. Comput. Fluid Mech. 12, 1, 104-116.

Wang W., Zheng D., Sheng G., Zhang Q., Su Y. (2017) A review of stimulated reservoir volume characterization for multiple fractured horizontal well in unconventional reservoirs, $A d v$. Geo-Energy Res. 1, 1, 54-63.

Warren J.E., Root P.J. (1963) The behavior of naturally fractured reservoirs, SPE J. 3, 3, 245-255.

Yao Y., Wu Y., Zhang R. (2012) The transient flow analysis of fluid in a fractal, double-porosity reservoir, Transp. Porous Media 94, 1, 175-187.

Ye W., Wang X., Cao C., Yu W. (2019) A fractal model for threshold pressure gradient of tight oil reservoirs, J. Pet. Sci. Eng. 179, 427-431.

Yin S., Xie R., Ding W., Shan Y., Zhou W. (2017) Influences of fractal characteristics of reservoir rocks on permeability, Lithologic Reserv. 29, 4, 81-90.

Yu B., Cheng P. (2002) A fractal permeability model for bidispersed porous media, Int. J. Heat Mass Transf. 45, 14, 2983-2993.

Yun M., Yu B., Cai J. (2009) Analysis of seepage characters in fractal porous media, Int. J. Heat Mass Transf. 52, 13, 32723278 .

Zhou D., Ge J., Li Y., Cai Y. (2000) Establishment of interporosity flow function of complex fractured reservoirs, OGRT 7, 2, 30-32.

Zimmerman R.W., Chen G., Hadgu T., Bodvarsson G.S. (1993) A numerical dual-porosity model with semianalytical treatment of fracture/matrix flow, Water Resour. Res. 29, 7, 21272137. 


\section{Appendix A}

\section{Derivation of 1D fractal pressure diffusion equation}

Supposing matrix has been surrounded by two fractures (Fig. 3), equation (A.1) for 1D matrix fractal pressure diffusion equation (Kong et al., 2007):

$$
\frac{\partial^{2} P}{\partial x^{2}}+\frac{1-D_{T}}{x} \frac{\partial P}{\partial x}=\frac{\phi_{a o} \mu c}{K_{a}}\left(\frac{x}{\lambda_{\max }}\right)^{2\left(D_{T}-1\right)} \frac{\partial P}{\partial t} .
$$

Let $W=P-P_{f}$, and initial and boundary conditions can be rewritten as:

$$
\begin{array}{cl}
W=P_{0}-P_{f}, \quad-L / 2 \leq x \leq L / 2, \quad t=0 \\
W=0, \quad x= \pm L / 2, \quad t>0 .
\end{array}
$$

Substituting equation (A.2) into equation (A.1), it yields:

$$
\frac{\partial^{2} W}{\partial x^{2}}+\frac{1-D_{T}}{x} \frac{\partial W}{\partial x}=\frac{\phi_{a \circ} \mu c}{K_{a}}\left(\frac{x}{\lambda_{\max }}\right)^{2\left(D_{T}-1\right)} \frac{\partial W}{\partial t} .
$$

Using separation variable method, it must have:

$$
W=u(x) \exp \left(-\frac{1}{M} \alpha^{2} t\right),
$$

where $M=\frac{\phi_{a o} \mu c}{K_{a} \lambda_{\max }^{2\left(D_{T}-1\right)}}$.

Substituting equation (A.4) into equation (A.3), we can obtain:

$$
\frac{\partial^{2} u}{\partial x^{2}}+\frac{1-D_{T}}{x} \frac{\partial u}{\partial x}+\alpha^{2} x^{2\left(D_{T}-1\right)} u=0 .
$$

According to the solution method of Bessel function, the solution of equation (A.5) can be obtained:

$$
\mu=\sum_{m=1}^{\infty}\left[A_{m} \sin \left(\frac{\alpha_{m} x^{D_{T}}}{D_{T}}\right)+B_{m} \cos \left(\frac{\alpha_{m} x^{D_{T}}}{D_{T}}\right)\right] .
$$

Substituting equation (A.6) into equation (A.4), it yields:

$$
W=\sum_{m=1}^{\infty}\left[A_{m} \sin \left(\frac{\alpha_{m} x^{D_{T}}}{D_{T}}\right)+B_{m} \cos \left(\frac{\alpha_{m} x^{D_{T}}}{D_{T}}\right)\right] \exp \left(-\frac{1}{M} \alpha_{m}^{2} t\right) .
$$

Combining equations (A.2) with (A.7), we can obtain:

$$
\begin{array}{r}
\sum_{m=1}^{\infty}\left[A_{m} \sin \left(\frac{\alpha_{m} L^{D_{T}}}{2^{D_{T}} D_{T}}\right)+B_{m} \cos \left(\frac{\alpha_{m} L^{D_{T}}}{2^{D_{T}} D_{T}}\right)\right] \exp \left(-\frac{1}{M} \alpha_{m}^{2} t\right)=0, \\
\text { (A.8a) } \\
\sum_{m=1}^{\infty}\left[-A_{m} \sin \left(\frac{\alpha_{m} L^{D_{T}}}{2^{D_{T}} D_{T}}\right)+B_{m} \cos \left(\frac{\alpha_{m} L^{D_{T}}}{2^{D_{T}} D_{T}}\right)\right] \exp \left(-\frac{1}{M} \alpha_{m}^{2} t\right)=0,
\end{array}
$$

$$
\sum_{m=1}^{\infty}\left[A_{m} \sin \left(\frac{\alpha_{m} x^{D_{T}}}{D_{T}}\right)+B_{m} \cos \left(\frac{\alpha_{m} x^{D_{T}}}{D_{T}}\right)\right]=P_{0}-P_{f} .
$$

Consequently, the solutions of equations (A.8a) and (A.8b) can be expressed as:

$$
A_{m}=0, \alpha_{m}=\frac{2^{D_{T}-1}(2 n+1) \pi D_{T}}{L^{D_{T}}} .
$$

Substituting equation (A.9) into equation (A.8c), equation (A.8c) can be rewritten as:

$$
P_{0}-P_{f}=\sum_{m=1}^{\infty} B_{m} \cos \left(\frac{\alpha_{m} x^{D_{T}}}{D_{T}}\right) .
$$

Both sides of equation (A.10) are multiplied by $x^{D_{T}-1} \cos \left(\frac{\alpha_{m} x_{T}}{D_{T}}\right)$ and integrated with respect to $x$. Then, the value of $B_{m}$ can be obtained as:

$$
\begin{aligned}
B_{m}= & \frac{1}{\int_{-L / 2}^{L / 2} x^{D_{T}-1} \cos ^{2}\left(\frac{\alpha_{m} x^{D_{T}}}{D_{T}}\right) \mathrm{d} x} \\
& \times \int_{-L / 2}^{L / 2}\left(P_{0}-P_{f}\right) x^{D_{T}-1} \cos \left(\frac{\alpha_{m} x^{D_{T}}}{D_{T}}\right) \mathrm{d} x \\
= & (-1)^{n} \frac{4\left(P_{0}-P_{f}\right)}{(2 n+1) \pi} .
\end{aligned}
$$

Substituting equations (A.9) and (A.11) into equation (A.7), thus, equation (A.7) becomes:

$$
\begin{aligned}
W= & P-P_{f}=\sum_{n=0}^{\infty}(-1)^{n} \frac{4\left(P_{0}-P_{f}\right)}{(2 n+1) \pi} \cos \left[\frac{(2 n+1) \pi}{2} \frac{x^{D_{T}}}{(L / 2)^{D_{T}}}\right] \\
& \times \exp \left[-\frac{1}{M} \frac{2^{2 D_{T}-2}(2 n+1)^{2} \pi^{2} D_{T}^{2}}{L^{2 D_{T}}} t\right] .
\end{aligned}
$$

Equation (A.12) can be converted to:

$$
\begin{aligned}
P= & P_{f}+\sum_{n=0}^{\infty}(-1)^{n} \frac{4\left(P_{0}-P_{f}\right)}{(2 n+1) \pi} \cos \left[\frac{(2 n+1) \pi}{2} \frac{x^{D_{T}}}{(L / 2)^{D_{T}}}\right] \\
& \times \exp \left[-\frac{1}{M} \frac{2^{2 D_{T}-2}(2 n+1)^{2} \pi^{2} D_{T}^{2}}{L^{2 D_{T}}} t\right] .
\end{aligned}
$$

Assuming that the area of matrix $A_{\text {av }}$ is equal to $h x, h$ is the height which is constant, and $x$ is distance. The average pressure of the matrix is as follows:

$$
\bar{P}=\frac{1}{A_{a v}} \int_{-L / 2}^{L / 2} P \mathrm{~d} A_{a v} .
$$

Substituting equation (A.13) into equation (A.14), it yields:

$$
\begin{aligned}
\bar{P}= & P_{f}+\sum_{n=0}^{\infty} \frac{(-1)^{n} 8\left(P_{0}-P_{f}\right)}{L \pi^{2}(2 n+1)^{2}} \\
& \times \delta \exp \left[-\frac{1}{M} \frac{2^{2 D_{T}-2}(2 n+1)^{2} \pi^{2} D_{T}^{2}}{L^{2 D_{T}}} t\right],
\end{aligned}
$$


where $\delta=\int_{-L / 2}^{L / 2} \cos \left[\frac{(2 n+1) \pi}{2} \frac{x^{D_{T}}}{(L / 2)^{D_{T}}}\right] \frac{1}{D_{T}}\left(\frac{x}{L / 2}\right)^{1-D_{T}} d\left[\frac{(2 n+1) \pi}{2} \frac{x^{D_{T}}}{(L / 2)^{D_{T}}}\right]$. Both sides of equation (A.15) are subtracted by $P_{0}$ and then divided by $P_{f}-P_{0}$. Thus, equation (A.15) can be expressed as:

$$
\begin{aligned}
\frac{\bar{P}-P_{0}}{P_{f}-P_{o}}= & 1-\sum_{n=0}^{\infty} \frac{(-1)^{n} 8 \times \mathrm{e}^{(2 n+1)^{2}}}{L \pi^{2}(2 n+1)^{2}} \\
& \times \delta \exp \left(-\frac{1}{M} \frac{2^{2 D_{T}-2} \pi^{2} D_{T}^{2}}{L^{2 D_{T}}} t\right) .
\end{aligned}
$$

\section{Appendix B}

\section{Derivation of 2D and 3D fractal pressure diffusion equation}

The matrix fractal pressure diffusion equation is given by (Kong et al., 2007):

$$
\frac{\partial^{2} P}{\partial r^{2}}+\frac{\xi-D_{T}}{r} \frac{\partial P}{\partial r}=\frac{\phi_{a o} \mu c}{K_{a}}\left(\frac{r}{\lambda_{\max }}\right)^{2\left(D_{T}-1\right)} \frac{\partial P}{\partial t},
$$

where $\xi$ corresponds to 2D flow and 3D flow, which are 2 and 3 , respectively.

Let $G=P-P_{f}$, and initial and boundary conditions can be rewritten as:

$$
\begin{gathered}
G=P_{0}-P_{f}, \quad 0 \leq r \leq R, \quad t=0 \\
G=0, \quad r=R, \quad t>0 .
\end{gathered}
$$

Substituting equation (B.2) into equation (B.1), it yields:

$$
\frac{\partial^{2} G}{\partial r^{2}}+\frac{\xi-D_{T}}{r} \frac{\partial G}{\partial r}=\frac{\phi_{a o} \mu c}{K_{a}}\left(\frac{r}{\lambda_{\max }}\right)^{2\left(D_{T}-1\right)} \frac{\partial G}{\partial t} .
$$

Using separation variable method, we can see that:

$$
G=w(r) \exp \left(-\frac{1}{M} \beta^{2} t\right)
$$

Substituting equation (B.4) into equation (B.3), we can obtain:

$$
\frac{\partial^{2} w}{\partial r^{2}}+\frac{\xi-D_{T}}{r} \frac{\partial w}{\partial r}=\beta^{2} r^{2\left(D_{T}-1\right)} \frac{\partial w}{\partial t} .
$$

According to the solution method of Bessel function, the solution of equation (B.5) can be obtained:

$$
\begin{aligned}
w & =r^{\frac{D_{T}+1-\xi}{2}} \sum_{m=1}^{\infty} \\
& \times\left[c_{m} J_{v}\left(\frac{\beta_{m} r^{D_{T}}}{D_{T}}\right)+b_{m} \frac{\cos (v \pi) J_{v}\left(\frac{\beta_{m} r^{D_{T}}}{D_{T}}\right)-J_{-v}\left(\frac{\beta_{m} r^{D_{T}}}{D_{T}}\right)}{\sin (v \pi)}\right],
\end{aligned}
$$

where $v=\frac{\left|D_{T}+1-\xi\right|}{2 D_{T}}, v$ corresponds to $2 \mathrm{D}$ flow and 3D flow, which are $v_{2}=\frac{D_{T}-1}{2 D_{T}}$ and $v_{3}=\frac{\left|D_{T}-2\right|}{2 D_{T}}$, respectively.

Substituting equation (B.6) into equation (B.4), it yields:

$$
\begin{aligned}
G & =r^{\frac{D_{T+1-\xi}}{2}} \sum_{m=1}^{\infty}\left[c_{m} J_{v}\left(\frac{\beta_{m} r^{D_{T}}}{D_{T}}\right)\right. \\
& \left.+b_{m} \frac{\cos (v \pi) J_{v}\left(\frac{\beta_{m} r^{D_{T}}}{D_{T}}\right)-J_{-v}\left(\frac{\beta_{m} r^{D_{T}}}{D_{T}}\right)}{\sin (v \pi)}\right] \exp \left(-\frac{1}{M} \beta_{m}^{2} t\right) .
\end{aligned}
$$

Combining equations (B.2) and (B.7), we can obtain:

$$
\begin{array}{r}
R^{\frac{D_{T}+1-\xi}{2} \sum_{m=1}^{\infty}}\left[c_{m} J_{v}\left(\frac{\beta_{m} R^{D_{T}}}{D_{T}}\right)+b_{m} \frac{\cos (v \pi) J_{v}\left(\frac{\beta_{m} R^{D_{T}}}{D_{T}}\right)-J_{-v}\left(\frac{\beta_{m} R^{D_{T}}}{D_{T}}\right)}{\sin (v \pi)}\right] \\
\times \exp \left(-\frac{1}{M} \beta_{m}^{2} t\right)=0
\end{array}
$$

$$
\begin{aligned}
r^{\frac{D_{T}+1-\xi}{2}} \sum_{m=1}^{\infty}\left[c_{m} J_{v}\left(\frac{\beta_{m} r^{D_{T}}}{D_{T}}\right)\right. & \left.+b_{m} \frac{\cos (v \pi) J_{v}\left(\frac{\beta_{m} r_{T}}{D_{T}}\right)-J_{-v}\left(\frac{\beta_{m} r_{T}}{D_{T}}\right)}{\sin (v \pi)}\right] \\
& =P_{0}-P_{f} .
\end{aligned}
$$

Equation (B.8b) can be converted to:

$$
\begin{gathered}
P_{0}-P_{f}=r^{\frac{D_{T}+1-\xi}{2}} \sum_{m=1}^{\infty} J_{v}\left(\frac{\beta_{m} r^{D_{T}}}{D_{T}}\right) \times\left[c_{m}+b_{m} \frac{\cos (v \pi)}{\sin (v \pi)}-b_{m} \frac{J_{-v}\left(\frac{\beta_{m} r^{D_{T}}}{D_{T}}\right)}{J_{v}\left(\frac{\beta_{m} r_{T}}{D_{T}}\right)}\right] \\
=r^{\frac{D_{T}+1-\xi}{2}} \sum_{m=1}^{\infty} J_{v}\left(\frac{\beta_{m} r^{D_{T}}}{D_{T}}\right) \\
\times\left[c_{m}+b_{m} \frac{\cos (v \pi)}{\sin (v \pi)}-b_{m}\left(\frac{\beta_{m} r^{D_{T}}}{2 D_{T}}\right)^{-2 v} \frac{\Gamma(m+1+v)}{\Gamma(m+1-v)}\right] . \quad \text { (B.9) }
\end{gathered}
$$

In equation (B.9), when $r \rightarrow 0$, we have $\left(\frac{\beta_{m} r_{T}}{2 D_{T}}\right)^{-2 v} \rightarrow \infty$, but $P_{0}-P_{f}<<\infty$, so we can obtain:

$$
b_{m}=0 \text {. }
$$

Then, equation (B.9) can be reduced to:

$$
P_{0}-P_{f}=r^{\frac{D_{T}+1-\xi}{2}} \sum_{m=1}^{\infty} c_{m} J_{v}\left(\frac{\beta_{m} r^{D_{T}}}{D_{T}}\right) .
$$

Substituting equation (B.10) into equation (B.8a) yields:

$$
R^{\frac{D_{T}+1-\xi}{2}} \sum_{m=1}^{\infty}\left[c_{m} J_{v}\left(\frac{\beta_{m} R^{D_{T}}}{D_{T}}\right)\right] \exp \left(-\frac{1}{M} \beta_{m}^{2} t\right)=0 .
$$

In equation (B.12), due to $R^{\frac{D_{T}+1-\xi}{2}} \neq 0$ and $\exp \left(-\frac{1}{M} \beta_{m}^{2} t\right) \neq 0$, so we can obtain: 


$$
\begin{gathered}
J_{v}\left(\frac{\beta_{m} R^{D_{T}}}{D_{T}}\right)=J_{v}\left(I_{m}\right)=0 \\
\text { so } \quad \beta_{m}=\frac{D_{T} I_{m}}{R^{D_{T}}}
\end{gathered}
$$

where $J_{v}(x)$ is the Bessel function, $v$ is the order of Bessel function, $I_{m}$ is the positive zero point of Bessel function (the solution code of $I_{m}$ is shown in Appendix $\mathrm{C}$ ), it depends on the value of $v, I_{m}$ corresponds to $2 \mathrm{D}$ flow and $3 \mathrm{D}$ flow, which are $I_{1}$ and $I_{2}$, respectively.

Both sides of equation (B.12) are multiplied by $\frac{r^{D_{T}}}{D_{T}} J_{v}\left(\frac{\beta_{m} r^{D_{T}}}{D_{T}}\right)$ and integrated with respect to $x$. Then, the value of $c_{m}$ can be obtained as:

$$
c_{m}=\frac{2\left(P_{0}-P_{f}\right)}{J_{v-1}\left(\frac{\beta_{m} R^{D_{T}}}{D_{T}}\right)}\left[-D_{T}^{-v}\left(\frac{R^{D_{T}}}{D_{T}}\right)^{-v-1} \beta_{m}^{v-2}\right] .
$$

Substituting equations (B.10), (B.13) and (B.14) into equation (B.7), thus, equation (B.7) becomes:

$G=P-P_{f}=r^{\frac{D_{T}+1-\xi}{2}} \sum_{m=1}^{\infty} \frac{2\left(P_{f}-P_{0}\right)}{J_{v-1}\left(I_{m}\right)} D_{T}{ }^{-v}\left(\frac{R^{D_{T}}}{D_{T}}\right)^{-v-1}$

$$
\times\left(\frac{D_{T} I_{m}}{R^{D_{T}}}\right)^{v-2} J_{v}\left(\frac{r^{D_{T}} I_{m}}{R^{D_{T}}}\right) \exp \left[-\frac{1}{M}\left(\frac{D_{T} I_{m}}{R^{D_{T}}}\right)^{2} t\right] .
$$

Equation (B.15) can be converted to:

$$
\begin{aligned}
P=P_{f}+ & r^{\frac{D_{T}+1-\xi}{2}} \sum_{m=1}^{\infty} \frac{2\left(P_{f}-P_{0}\right)}{J_{v-1}\left(I_{m}\right)} D_{T}^{-v}\left(\frac{R^{D_{T}}}{D_{T}}\right)^{-v-1}\left(\frac{D_{T} I_{m}}{R^{D_{T}}}\right)^{v-2} \\
& \times J_{v}\left(\frac{r^{D_{T}} I_{m}}{R^{D_{T}}}\right) \exp \left[-\frac{1}{M}\left(\frac{D_{T} I_{m}}{R^{D_{T}}}\right)^{2} t\right]
\end{aligned}
$$

\section{Appendix C}

\section{Solution code of the positive zero of Bessel function}

In this paper, we need to get the positive zero of Bessel function which is solved by MATLAB software. The specific code is as follows:

clear, clc;

format long

$\mathrm{x}=(0: 0.2: 1)^{\prime}$;

y_0=fzero $(@(x) \operatorname{besselj}(0.045, x), 3)$; 